\title{
In-line Spectral Interferometry in Shortwave-Infrared Laser Filaments in Air
}

\author{
Xiaoming Ren, ${ }^{1}$ Yang Wang, ${ }^{1}$ Zenghu Chang, ${ }^{1}$ James Welch, ${ }^{2}$ Aaron Bernstein, ${ }^{2}$ \\ Michael Downer, ${ }^{2}$ Jeffrey Brown, ${ }^{3,4}$ Mette Gaarde, ${ }^{3}$ Arnaud Couairon, ${ }^{4}$ \\ Miroslav Kolesik, ${ }^{5}$ and Pavel Polynkin,"* \\ ${ }^{1}$ Department of Physics and CREOL, The College of Optics and Photonics, \\ University of Central Florida, Orlando, Florida 21816, USA \\ ${ }^{2}$ Department of Physics, University of Texas at Austin, Austin, Texas 78712, USA \\ ${ }^{3}$ Department of Physics and Astronomy, Louisiana State University, Baton Rouge, Louisiana 70803, USA \\ ${ }^{4}$ CPHT, CNRS, Ecole Polytechnique, IP Paris, F-91128 Palaiseau, France \\ ${ }^{5}$ College of Optical Sciences, The University of Arizona, Tucson, Arizona 85721, USA
}

(Received 24 May 2019; revised manuscript received 22 August 2019; published 26 November 2019)

\begin{abstract}
We investigate the nonlinear propagation of intense, two-cycle, carrier-envelope phase (CEP) stable laser pulses at $1.7 \mu \mathrm{m}$ center wavelength in air. We observe CEP-dependent spectral interference in the visible part of the forward-propagating white light generated on propagation. The effect is robust against large fluctuations of the input pulse energy. This robustness is enabled by rigid clamping of both the peak optical field and the phase of the propagating waveform, which has been revealed by numerical simulations. The CEP locking can enhance the yield of the CEP-dependent strong-field processes in gaseous media with long-wavelength drivers, while the observed spectral interference enables single-shot, stand-off CEP metrology in the atmosphere.
\end{abstract}

DOI: 10.1103/PhysRevLett.123.223203

Nonlinear self-channeling (filamentation) of intense, ultrashort laser pulses in gases allows us to project strong-field phenomena to extended standoff distances and enables various potential applications ranging from remote sensing to lightning protection [1-3]. The majority of past studies of filamentation used ultrafast Ti:sapphire lasers operating in the near-infrared (NIR) and generating laser pulses with durations from tens to hundreds of femtoseconds. Recent progress in the ultrafast laser technology pushes these investigations into new regimes.

Spectral coverage of filamentation studies has been recently extended to short-wave infrared (SWIR) [4,5], midwave infrared (MWIR) [6,7], and long-wave infrared (LWIR) [8] spectral domains. It has been argued that, at longer wavelengths, the physics governing filamentary propagation, as well as the properties of filaments, are expected to be different from those in the familiar NIR regime [9]. Trace constituents of air, such as $\mathrm{CO}_{2}$ and $\mathrm{H}_{2} \mathrm{O}$, introduce spectral windows of anomalous dispersion and loss, enabling solitonlike propagation [10] and new mechanisms of nonlinear absorption [11]. Multiple odd harmonics of the infrared driver can be readily detected and analyzed [12].

The use of few-cycle pulses in the studies of filamentation has been scarce $[13,14]$. Filamentation in noble gases has been shown to be accompanied by compression of NIR laser pulses from tens of femtoseconds well into the sub-ten femtosecond range $[15,16]$. The CEP of the resulting few-cycle optical waveforms has been robust against percent-level fluctuations of the input pulse energy [17]. On the other hand, in a computational study of filamentation-based compression of laser pulses at $800 \mathrm{~nm}$ and $2 \mu \mathrm{m}$ [18], percent-level pulse-energy fluctuations have been detrimental to the preservation of the CEP coherence.

Here, we report the results of experiments and numerical simulations on the filamentary propagation of two-cycle laser pulses at $1.7 \mu \mathrm{m}$ center wavelength in air. We observe CEP-dependent interference in the spectrum of the generated visible white light. The effect is robust against large pulse-energy fluctuations, indicating that SWIR filamentation preserves the intrapulse coherence [19]. Numerical simulations reveal rigid carrier-level clamping of both the peak optical field and the CEP of the entire propagating waveform. As a result, the complete loss of phase that has been argued to universally accompany filamentation [20] does not occur in our experiments. The CEP clamping and the spectral interference effects are expected to universally occur in the SWIR and LWIR filamentation in different gases, enabled by the inherently nonperturbative, carrierlevel strong-field physics with long-wavelength drivers. The preservation of the CEP over the extended, highly nonlinear propagation can enhance the yield of various strong-field processes, such as high-harmonic generation and the production of energetic electrons. The observed spectral interference can be used for the single-shot characterization of the CEP in filamentation with CEP-unstable laser systems, enabling standoff CEP metrology in the atmosphere. 
Our experiments made use of the SWIR optical parametric chirped-pulse amplification system that generated laser pulses at $1.7 \mu \mathrm{m}$ center wavelength, with $11 \mathrm{fs}$ duration at FWHM, at the pulse repetition rate of $1 \mathrm{kHz}$ [21]. The maximum pulse energy was $1.4 \mathrm{~mJ}$. The flat-top, $12 \mathrm{~mm}$-diameter laser beam was weakly focused in air, using a gold-coated concave mirror with the focal length of $2 \mathrm{~m}$

The longitudinal distribution of plasma density in the SWIR filament was measured using a capacitive plasma probe [22] that was separately calibrated using a NIR filament generated under specific conditions, for which the peak plasma density has been independently measured on the absolute scale [23]. Measurable plasma started at $\sim 10 \mathrm{~cm}$ before the geometrical focal plane of the focusing mirror and extended over $\sim 15 \mathrm{~cm}$. The transverse size of the filament core was visualized by making a single-shot burn on a gold-coated silicon wafer [24]. Based on these measurements, we roughly estimated the peak plasma density in the SWIR filament at $\sim 10^{22} \mathrm{~m}^{-3}$. This estimate is consistent with the measured energy loss of $\sim 2 \%$ that the laser pulse experienced on propagation through the filamentation zone. The confocal parameter (twice the Rayleigh range) corresponding to the transverse size of the filament core was about $15 \mathrm{~mm}$. The measured length of the plasma channel was about 10 times larger, indicating the occurrence of the nonlinear self-channeling.

Spectra of the forward-propagating white light generated by the filament have been analyzed, in the far field, with the aid of the angularly resolving imaging spectrometer [25]. The two-dimensional spectral map at the output plane of the spectrometer has the wavelength components of the emission resolved horizontally and the angles of emission, with respect to the beam axis, resolved vertically. The resulting $\theta-\lambda$ spectra reveal emission features that may be hidden in the conventional spectra that are integrated over all emission angles [26]. The CCD sensor used to record the spectra was sensitive in the wavelength range from $300 \mathrm{~nm}$ to $1 \mu \mathrm{m}$, which covered the third harmonic of the SWIR driver. Emission above $\sim 650 \mathrm{~nm}$ was strongly attenuated by a color-glass filter.

Two examples of $\theta-\lambda$ spectra corresponding to two specific values of the CEP, relative to an arbitrary constant phase, are shown in Fig. 1. The evident CEP-dependent feature is the appearing and disappearing spectral hole in the on-axis emission at around $525 \mathrm{~nm}$, which is close to the wavelength of the third harmonic of the fundamental SWIR pulse. An examination of the movie showing evolving $\theta-\lambda$ spectrum at a gradually advancing value of the CEP (available in the Supplemental Material [27]) reveals that the spectral hole is a part of an about two period-long spectral beat pattern spanning from $\sim 500$ to $\sim 575 \mathrm{~nm}$. The pattern is $\pi$ periodic in the CEP, consistently with two pulses with the CEP values different by $\pi$ being physically indistinguishable. Similar spectral interference

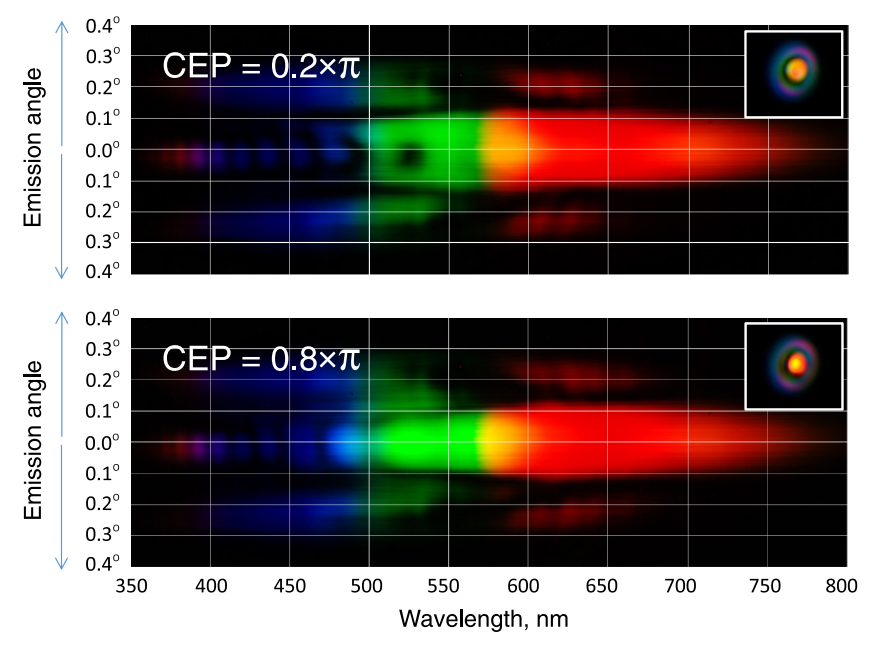

FIG. 1. Measured $\theta-\lambda$ spectra for two specific values of the CEP that correspond to the most dissimilar spectra. The images are integrated over 750 laser shots. The specified CEP values are relative to an arbitrary constant number. Colors in these images correspond to actual colors, as perceived by the human eye. The insets show images of the corresponding beam patterns, were emissions at different angles are strongly overlapping. A movie composed of the $\theta-\lambda$ spectra for the gradually advancing value of the CEP is available in Ref. [27].

has been previously reported in the supercontinuum generation with ultrashort SWIR laser pulses in transparent solids [28,29], where it occurred at much lower pulse energy and with much lesser contributions from ionization and self-channeling of the laser beam.

The two spectra shown in Fig. 1, as well as all frames in the movie of the evolving spectrum in Ref. [27], have been averaged over 750 laser shots. The shot-to-shot pulseenergy fluctuations of the SWIR driver laser are about $2.5 \%$ rms. It appears counterintuitive that the multishot averaging has not smeared out the contrast of the spectral interference pattern, given that the pulse experienced complex, highly nonlinear propagation through the filamentation zone. The reason for this robustness was revealed by numerical simulations of our experiments that we discuss next.

Our numerical simulations utilized an axially symmetric, nonparaxial, unidirectional propagator for the linearly polarized optical field [30]. The third-harmonic content of the pulse is populated by the action of two additive source terms in the field propagator: the nonlinear polarization due to the Kerr effect and the time-varying electric current. The latter is due to the carrier-level dependence of the strong-field ionization of air molecules (the Brunel current, [31]) and includes the contribution representing energy loss to ionization [32].

Ionization rates for oxygen and nitrogen are calculated according to the Perelomov, Popov, and Terent'ev (PPT) formalism [33], which is modified by using the carrierlevel "intensity" of the optical field $I(t) \equiv \epsilon_{0} c n_{0} E^{2}(t)$. 


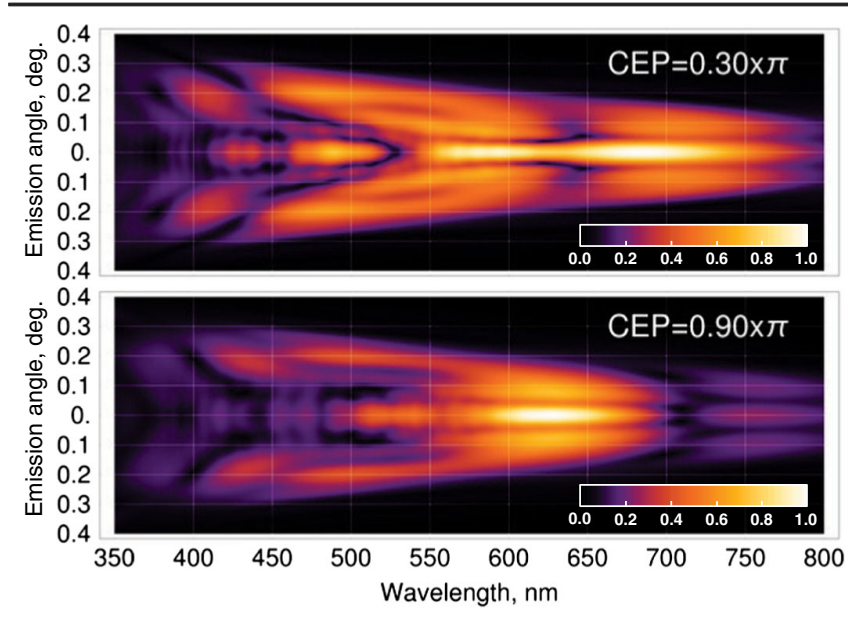

FIG. 2. Numerical results for the $\theta-\lambda$ spectra, shown on an arbitrary linear scale, for two particular values of the input CEP that correspond to the most dissimilar spectra. The color palette is chosen to highlight the generated spectral features. A movie composed of the $\theta-\lambda$ spectra for gradually advancing values of the CEP is available in Ref. [27].

Here $n_{0}$ is a constant, spectrally averaged value of the linear refractive index of air and $E(t)$ is real, instantaneous electric field. The prefactor in the PPT rate is modified as discussed in Ref. [34]. For generality, dispersion and linear absorption of air are computed according to the HITRAN database [35], accounting for 50\% humidity and 500 ppm concentration of $\mathrm{CO}_{2}$. Under our experimental conditions, the water and $\mathrm{CO}_{2}$ contents have negligible influence on the simulation results, although they are expected to make observable differences for long-range propagation. The duration, spectrum, beam shape, and focusing conditions for the input pulse match those used in the experiment.

Numerical simulations using our model have been conducted for the input CEP values in the interval from 0 to $\pi$, with the step of $0.05 \times \pi$. As discussed above, the $\theta-\lambda$ spectra in the interval of the CEPs from $\pi$ to $2 \pi$ identically reproduce those in the interval from 0 to $\pi$. The field was propagated from the focusing mirror at $0 \mathrm{~cm}$, where the initial CEP of the laser pulse was defined, to the longitudinal coordinate of $250 \mathrm{~cm}$, which is $50 \mathrm{~cm}$ after the geometrical focal plane of the mirror; the field at $250 \mathrm{~cm}$ is referred to as the output field. The absolute input CEP values in the experiment and simulation differ by a constant unknown phase.

Simulations reproduce the main experimentally observed features of the $\theta-\lambda$ spectra-the CEP-dependent spectral interference in the vicinity of the third harmonic, as well as the generation of the off-axis emission ring. The two extreme cases-with and without a pronounced spectral hole in the on-axis emission near third harmonic of the driver, shown in Fig. 2, correspond to the initial CEPs of $0.30 \times \pi$ and $0.90 \times \pi$, respectively. In the simulated spectra, emission above $\sim 650 \mathrm{~nm}$ is numerically attenuated, using

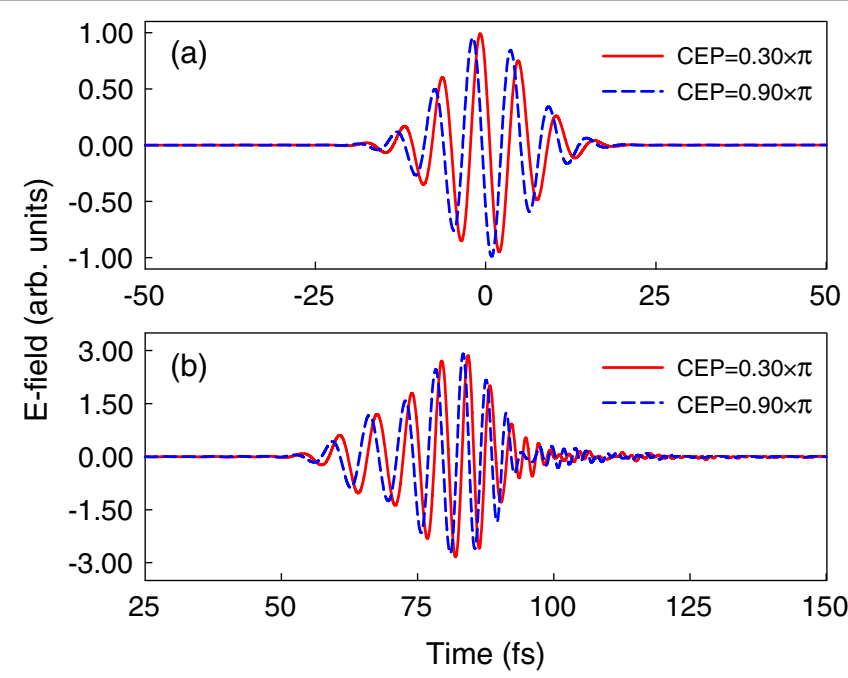

FIG. 3. (a) The simulated $E$-field waveforms for the two values of the CEP that correspond to the two output $\theta-\lambda$ spectra shown in Fig. 2. (b) Corresponding output $E$-field waveforms of the onaxis emission component. Input pulse energy is $1.40 \mathrm{~mJ}$. The overall CEP of the waveform is rigidly locked on passing through the filamentation zone.

the spectral transmission function of the color-glass filter used in the experiment.

The noticeable quantitative differences between the experimental and numerically simulated $\theta-\lambda$ spectra are not unexpected, given the complexity of extended, highly nonlinear propagation of the laser pulse and the limited accuracy to which the ionization of air with few-cycle, SWIR laser pulses can be realistically computed. The sharper and richer structure of the simulated spectral patterns, compared to those recorded experimentally, is predominantly due to the much higher spectral resolution used in the simulation. Neither the numerical averaging over many laser shots with fluctuating pulse energy, nor varying the concentrations of the trace constituents of air have resulted in a better quantitative match between experiment and simulation.

Simulations allow for an in-depth examination of the pulse dynamics on the passage through the filamentation zone. A movie showing pulse evolution is available in the Supplemental Material [27]. This evolution is quite complex and results in the formation of a chirped pulse with the high-frequency content trailing behind the main pulse waveform. In Fig. 3, we show examples of the input and output $E$-field waveforms for the input pulse energy of $1.40 \mathrm{~mJ}$ and for the two specific values of the input CEP that correspond to the $\theta-\lambda$ spectra shown in Fig. 2 . Rigid preservation of the initial CEP throughout the highly nonlinear propagation is evident.

To elucidate the origin of the spectral interference effect, we isolate the third-harmonic $(3 \omega)$ content by numerical band-pass spectral filtering of the full output optical waveform, using a $100 \mathrm{~nm}$-wide filter function centered 


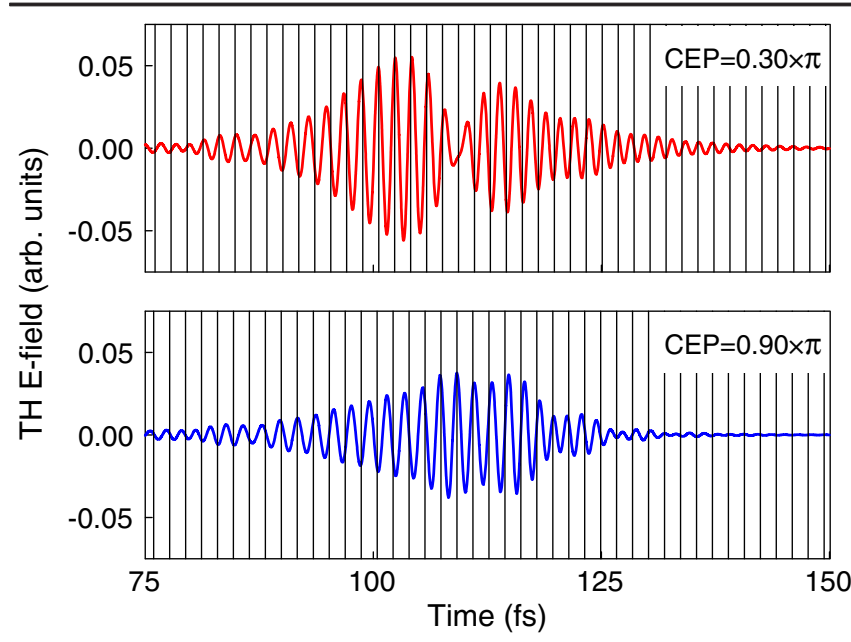

FIG. 4. The simulated third-harmonic content of the output temporal $E$-field waveforms. The vertical grids show the periodicity of the monochromatic wave at $525 \mathrm{~nm}$ wavelength, where the spectral hole is observed in the simulation. In the case of $\mathrm{CEP}=0.30 \times \pi$, the output waveform at the third harmonic consists of two distinct subpulses that are predominantly out of phase with each other. In the case of CEP $=0.90 \times \pi$, the subpulses in the third-harmonic waveform are largely in phase and strongly overlapping.

at $525 \mathrm{~nm}$ (the wavelength of the on-axis spectral hole observed in the simulation for the input CEP $=0.30 \times \pi$ ). The result is shown in Fig. 4, for the two specific values of the input CEP used in Figs. 2 and 3. As expected, the output $3 \omega$ waveform is strongly influenced by the input CEP. While the fundamental frequency content remains a single pulse on propagation for any CEP, which is evident in Fig. 3, the third-harmonic content, trailing behind the fundamental, consists of two overlapping subpulses. For the input CEP of $0.30 \times \pi$, the two third-harmonic subpulses are predominantly out of phase with each other, producing a pronounced spectral hole at $525 \mathrm{~nm}$. For the input CEP $=0.90 \times \pi$, the subpulses are predominantly in phase and strongly overlapping; they appear as a single, longer pulse, and the spectral hole at third harmonic is suppressed.

The presence of the two distinct third-harmonic pulses with the relative phase linked to the input CEP suggests that the $3 \omega$ content is populated in two stages, bearing the analogy with the conventional, solid-state $f-2 f$ interferometer, commonly used for single-shot CEP characterization [36]. The source terms predominantly responsible for the generation of third harmonic in these two stages carry different integer multiples of the original CEP. The groupvelocity dispersion (GVD) of plasma delays the third harmonic content generated in the first stage with respect to the leftover fundamental. The resulting $3 \omega$ subpulse trails behind the second $3 \omega$ subpulse generated later along the propagation path.

Note that clean separation between the sources responsible for the generation of the two subpulses at $3 \omega$ is not possible. However, even though the pulse dynamics are complex, they are pulse-to-pulse repeatable, resulting in the nonlinear generation of by products that robustly depend on the CEP of the input pulse. Since the GVD of plasma is predominantly responsible for the delay between the two third-harmonic subpulses, a longer filament and/or higher plasma density are expected to produce a more clear spectral interference pattern with denser interference fringes.

Both in the experiments and in the simulations, the differences between the CEP values corresponding to the most dissimilar spectra are different from $\pi / 2$, as would be the case for the $f-2 f$ interferometer, where spectral broadening, dispersive delay, and harmonic generation occur separately, in discrete optical elements. Here, the actions of different linear and nonlinear sources driving field evolution occur concurrently during propagation and are inherently mixed.

Following the logic of Refs. [37,38], we attempted to apply the first Born approximation to the computation of the output spectra. To that end, we used a truncated $E$ field, with the third-harmonic content numerically filtered out, for computing different source terms in the field propagatorthe Kerr nonlinearity, the Brunel current, and the nonlinear loss current. In this approximation, the high-frequency content, being weak in comparison with the content around the fundamental wavelength of the driver, is assumed to be generated exclusively by the nonlinear conversion of the fundamental, not influencing its own generation in an appreciable way. We found that the result of such an approximate computation of the spectrum around third harmonic grossly disagrees with the full simulation. Thus, the generation of the $3 \omega$ content in our experiment is nonperturbative, and the first Born approximation is not applicable to computing it.

It is remarkable how robust the complex and highly nonlinear pulse dynamics are against relatively large fluctuations of the energy of the incident SWIR laser pulse. This robustness is what preserves the experimentally observed spectral interference features through averaging over multiple laser shots with fluctuating energy. In Fig. 5, we show the complete and third-harmonic output waveforms for the input CEP value of $0.30 \times \pi$ and at two input pulse energies, different from one another by as much as 10\%. (In the experiment, the pulse-energy fluctuations were lower-about $2.5 \%$ rms.) The change of the complete waveform due to the energy difference is barely noticeableboth in amplitude and phase. The change of the $3 \omega$ content of the waveform is apparent but also not significant.

The preservation of the phase coherence observed in our experiments and simulations is the manifestation of the general trend of the transition from NIR to longer wavelengths: In the NIR, nonlinear optical interactions largely occur on the pulse-envelope level, with the carrier-level effects averaging out underneath the envelope. In the SWIR 

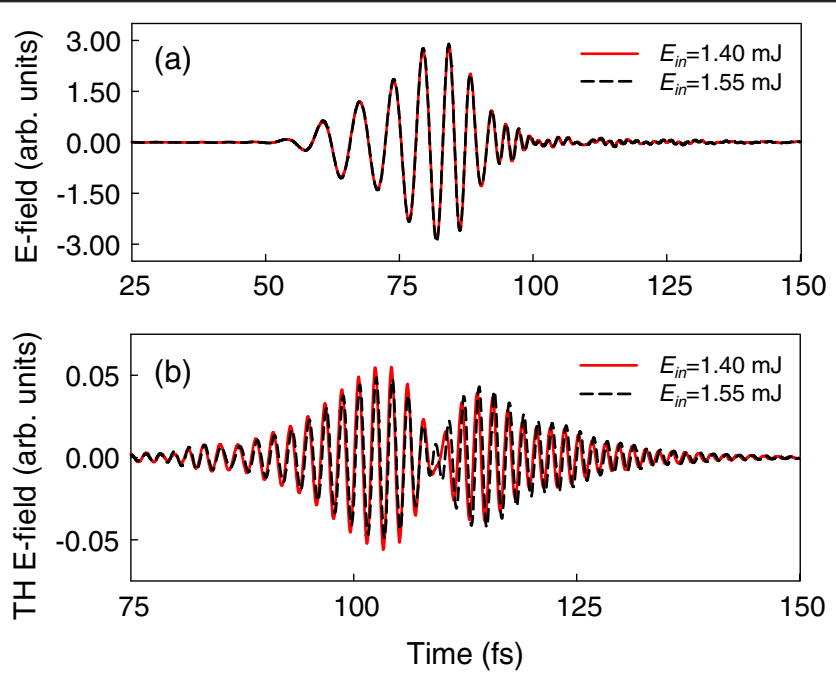

FIG. 5. The simulated complete (a) and third-harmonic (b) output fields, for the input CEP $=0.30 \times \pi$ and at two different energies of the input pulse, as specified in the legends. $10 \%$ energy variation causes only a very slight change of the output waveforms, both in amplitude and phase.

and longer wavelengths, on the other hand, the interactions are more quasistatic and follow the instantaneous field; subcycle physics emerges, and the overall pulse coherence survives highly nonlinear propagation.

In conclusion, we have investigated the nonlinear propagation of intense, two-cycle, CEP-stable, SWIR laser pulses at $1.7 \mu \mathrm{m}$ center wavelength in air. We observed rigid locking of the CEP of the optical waveform, propagating in the highly nonlinear regime, and the CEPdependent interference within the spectral content around the third harmonic of the SWIR driver, generated on propagation. Both the CEP locking and the spectral interference are robust against large pulse-to-pulse energy fluctuations. These effects are expected to universally occur in filamentation of long-wavelength, ultrashort-pulse laser beams in different gases. Numerical simulations reveal that the generation of white light that accompanies filamentation of long-wavelength, ultrashort laser pulses is a nonperturbative process, and the first-Born approximation is not applicable to adequately simulating it. The CEP locking over extended nonlinear propagation can enhance the yield of extreme nonlinear-optic conversion processes. The observed spectral interference enables single-shot measurements of the CEP of the ultrashort, long-wavelength laser pulses undergoing nonlinear self-channeling in air. This capability will be important in the long-range applications of the CEP-dependent strong-field phenomena, such as high-harmonic generation and the production of energetic electrons.

P. P. thanks A. Baltuska, L. Berge, A. Husakou, and M. Ivanov for helpful discussions. This material is based upon work supported by the U.S. Air Force Office of Scientific
Research under MURI Grant No. FA9550-16-1-0013. The contribution of M.K. was supported by the US AFOSR MURI Grant No. FA9550-16-1-0121.

*ppolynkin@optics.arizona.edu

[1] A. Couairon and A. Mysytowicz, Phys. Rep. 441, 47 (2007).

[2] L. Berge, S. Skupin, R. Nuter, J. Kasparian, and J.-P. Wolf, Rep. Prog. Phys. 70, 1633 (2007).

[3] S. L. Chin, Femtosecond Laser Filamentation (Springer, New York, 2010).

[4] Y. Bai, L. Song, R. Xu, C. Li, P. Liu, Z. Zeng, Z. Zhang, H. Lu, R. Li, and Z. Xu, Phys. Rev. Lett. 108, 255004 (2012).

[5] H. Liang, D. L. Weerawarne, P. Krogen, R. I. Grynko, C.-J. Lai, B. Shim, F. X. Kartner, and K.-H. Hong, Optica 3, 678 (2016).

[6] D. Kartashov, S. Alisauskas, A. Pugzlys, A. Voronin, A. Zheltikov, M. Petrarca, P. Bejot, J. Kasparian, J.-P. Wolf, and A. Baltuska, Opt. Lett. 37, 3456 (2012).

[7] A. V. Mitrofanov, A. A. Voronin, D. A. Sidorov-Biryukov, A. Pugzlys, E. A. Stepanov, G. Andriukaitis, T. Flory, S. Alisauskas, A. B. Fedotov, A. Baltuska, and A. M. Zheltikov, Sci. Rep. 5, 8368 (2015).

[8] S. Tochitsky, E. Welch, M. Polyanskiy, I. Pogorelsky, P. Panagiotopolous, M. Kolesik, E. M. Wright, S. W. Koch, J. V. Moloney, J. Pigeon, and C. Joshi, Nat. Photonics 13, 41 (2019).

[9] P. Panagiotopolous, P. Whalen, M. Kolesik, and J. V. Moloney, Nat. Photonics 9, 543 (2015).

[10] V. Shumakova, S. Alisauskas, P. Malevich, A. A. Voronin, A. V. Mitrofanov, D. A. Sidorov-Biryukov, A. M. Zheltikov, D. Kartashov, A. Baltuska, and A. Pugzlys, Opt. Lett. 44, 2173 (2019).

[11] N. A. Panov, D. E. Shipilo, V. A. Andreeva, O. G. Kosareva, A. M. Saletsky, H. Xu, and P. Polynkin, Phys. Rev. A 94, 041801(R) (2016).

[12] A. V. Mitrofanov, A. A. Voronin, S. I. Mitryukovskiy, D. A. Sidorov-Biryukov, A. Pugzlys, G. Andriukaitis, T. Flory, E. A. Stepanov, A. B. Fedotov, A. Baltuska, and A. M. Zheltikov, Opt. Lett. 40, 2068 (2015).

[13] D. E. Laban, W. C. Wallace, R. D. Glover, R. T. Sang, and D. Kielpinski, Opt. Lett. 35, 1653 (2010).

[14] X. Chen, P. Polynkin, and M. Kolesik, Opt. Lett. 38, 2017 (2013).

[15] C. P. Hauri, W. Kornelis, F. W. Helbing, A. Heinrich, A. Couairon, A. Mysyrowicz, J. Biegert, and U. Keller, Appl. Phys. B 79, 673 (2004).

[16] G. Stibenz, N. Zhavoronkov, and G. Steinmeyer, Opt. Lett. 31, 274 (2006).

[17] A. Guandalini, P. Eckle, M. Anscombe, P. Schlup, J. Biegert, and U. Keller, J. Phys. B 39, S257 (2006).

[18] L. Berge, C.-L. Soulez, C. Kohler, and S. Skupin, Appl. Phys. B 103, 563 (2011).

[19] N. Raabe, T. Feng, T. Witting, A. Demircan, C. Bree, and G. Steinmeyer, Phys. Rev. Lett. 119, 123901 (2017).

[20] B. Shim, S. E. Schrauth, A. L. Gaeta, M. Klein, and G. Fibich, Phys. Rev. Lett. 108, 043902 (2012).

[21] Y. Yin, J. Li, X. Ren, K. Zhao, Y. Wu, E. Cunningham, and Z. Chang, Opt. Lett. 41, 1142 (2016). 
[22] P. Polynkin, Appl. Phys. Lett. 101, 164102 (2012).

[23] Y.-H. Chen, S. Varma, T. M. Antonsen, and H. M. Milchberg, Phys. Rev. Lett. 105, 215005 (2010).

[24] X.-L. Liu, W. Cheng, M. Petrarca, and P. Polynkin, Opt. Lett. 41, 4751 (2016).

[25] D. Faccio, P. Di Trapani, S. Minardi, A. Bramati, F. Bragheri, C. Liberale, V. Degiorgio, A. Dubietis, and A. Matijosius, J. Opt. Soc. Am. B 22, 862 (2005).

[26] M. Kolesik, E. M. Wright, and J. V. Moloney, Opt. Express 13, 10729 (2005).

[27] See Supplemental Material at http://link.aps.org/ supplemental/10.1103/PhysRevLett.123.223203 for movies of the measured and simulated far-field $\theta-\lambda$ spectra with varying CEP of the input laser pulse, as well as for movies of the simulated evolution of the fundamental and third-harmonic $E$-field waveforms along the propagation path.

[28] J. Darginavičius, D. Majus, V. Jukna, N. Garejev, G. Valiulis, A. Couairon, and A. Dubietis, Opt. Express 21, 25210 (2013).
[29] C. Gong, J. Jiang, C. Li, L. Song, Z. Zeng, Y. Zheng, J. Miao, X. Ge, Y. Deng, R. Li, and Z. Xu, Opt. Express 21, 24120 (2013).

[30] M. Kolesik and J. V. Moloney, Phys. Rev. E 70, 036604 (2004).

[31] F. Brunel, J. Opt. Soc. Am. B 7, 521 (1990).

[32] P. Jurgens, B. Liewehr, B. Kruse, C. Peltz, D. Engel, A. Husakou, T. Witting, M. Ivanov, M. J. J. Vrakking, T. Fennel, and A. Mermillod-Blondin, arXiv:1905.05126.

[33] A. Perelomov, V. Popov, and M. Terent'ev, Sov. Phys. JETP 23, 924 (1966) [Zh. Eksp. Teor. Fiz. 50, 1393 (1966)].

[34] S. C. Rae and K. Burnett, Phys. Rev. A 46, 2077 (1992).

[35] https://hitran.org.

[36] A. Baltuska, M. Uiberacker, E. Goulielmakis, R. Kienberger, V. S. Yakovlev, T. Udem, T. W. Hansch, and F. Krausz, IEEE J. Selected Topics Quantum Electron. 9, 972 (2003).

[37] M. Kolesik, E. M. Wright, and J. V. Moloney, Opt. Lett. 32, 2816 (2007).

[38] J. M. Brown, A. Couairon, P. Polynkin, and M. B. Gaarde, J. Opt. Soc. Am. B 36, A105 (2019). 